\title{
Decision-Theoretic Personality-Based Reasoning about Turn-Taking Conflicts
}

Extended Abstract

\begin{abstract}
This paper outlines the use of an influence diagram for modeling turn-taking timing. In contrast to related works, our model focuses on an agent's personality and attitude towards the conversation partner. We also describe how this model is implemented in a first prototype application.
\end{abstract}

\section{CCS CONCEPTS}

- Human-centered computing $\rightarrow$ Systems and tools for interaction design; HCI theory, concepts and models;

\section{KEYWORDS}

human-agent interaction, personality modeling, interpersonal stance, turn taking conflicts, interruptions, decision-theoretic approach, influence diagram, Bayesian network

\section{ACM Reference Format:}

Kathrin Janowski and Elisabeth André. 2018. Decision-Theoretic PersonalityBased Reasoning about Turn-Taking Conflicts: Extended Abstract. In IVA '18: International Conference on Intelligent Virtual Agents (IVA '18), November 5-8, 2018, Sydney, NSW, Australia. ACM, New York, NY, USA, Article 4, 2 pages. https://doi.org/10.1145/3267851.3267899

\section{INTRODUCTION}

Talking computer-controlled agents are becoming more and more prevalent in our society. They may be disembodied voice assistants like Amazon's Alexa. They may be social robots, sitting on your living room table or roaming the stores. They may be virtual actors in simulated role play for cultural coaching or job interview training. All of them require appropriate floor management in order to interact with humans in a natural, intuitive and fluent manner. They need to know when they are allowed or expected to speak, when they have to yield their turn to the user, and when they have to stand their ground in order to deliver a crucial message. Furthermore, humans tend to attribute social characteristics to such characters, meaning that their behavior also needs to be consistent with the interpersonal dynamics required by the scenario.

The timing of a person's speech is closely related to their personality and their attitude towards the other participants. For instance,

IVA '18, November 5-8, 2018, Sydney, NSW, Australia @ 2018 Copyright held by the owner/author(s). ACM ISBN 978-1-4503-6013-5/18/11.

. This is the author's version of the work. It is posted here for your personal use. Not for redistribution. The definitive Version of Record was published at https://doi.org/10.1145/3267851.3267899

\author{
Elisabeth André \\ Human Centered Multimedia, Augsburg University \\ Augsburg, Germany \\ andre@informatik.uni-augsburg.de
}

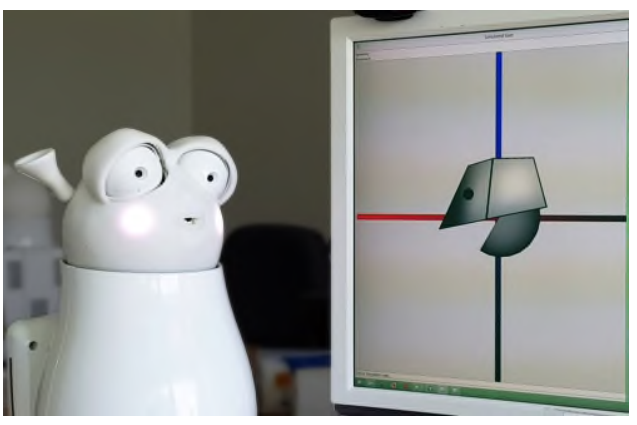

Figure 1: Reeti robot in conversation with PseudoBot agent.

dominant people are known to interrupt others more often [8]. Likewise, virtual agents who start speaking before the other party had finished have been rated as less agreeable than those who started afterwards [9], and those who are interrupted appear more dominant and less friendly when they continue speaking instead of backing down [2,9]. However, overlapping speech is not always a sign of dominance or unfriendliness [6]. Sometimes it can indicate enthusiasm and involvement, which are signs of good rapport and collaboration between the participants.

Consequently, conversational timing between agents and humans needs to be calculated from a wide range of interaction goals and influence factors. Further challenges arise from uncertainty caused by sensor noise or semantic ambiguities. One common answer to these problems is a decision-theoretic approach. For this, the system calculates the probabilities of achieving different outcomes, which in turn carry costs and benefits for the agent's goals. The expected utility of each available action is then used to find the most rewarding strategy. For example, Bohus and Horvitz [1] applied this approach to the turn-taking of a virtual quizmaster. While they did not model the agent's personality or attitude towards the players, they already accounted for uncertain observations and balanced the risk of overlapping speech against that of unnecessary silence. Conati [4] described a virtual butler which uses a dynamic decision network to infer the user's emotional state and its causes from noisy or incomplete inputs. This way the system actions can be scheduled for the time at which it causes the least harm or even improves the user's mood.

We adopt a similar approach to the works described above $[1,4]$, but focus on the impact which personality and interpersonal stance have on a conversational agent's turn-taking behavior. 


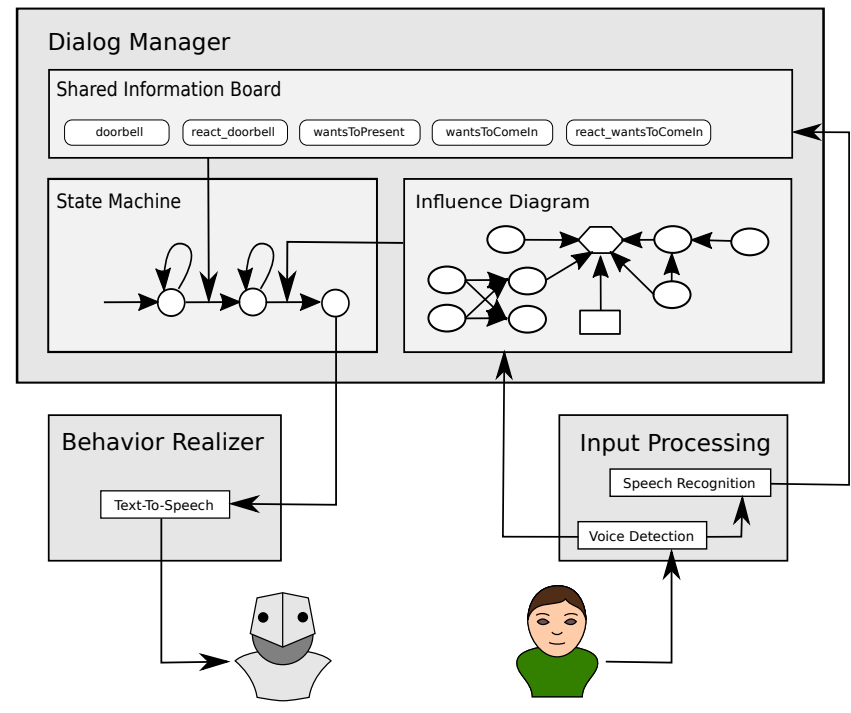

Figure 2: The architecture of our first prototype application.

\section{PROTOTYPE IMPLEMENTATION}

This section will describe the implementation of our first proof-ofconcept prototype. Figure 2 shows its most relevant components.

\subsection{Conversational Agents}

In order to test the computational model with noise-free, reproducible input, we chose to simulate conversations between two computer-controlled agents. ${ }^{1}$

The dialog manager uses a generic messaging protocol for connecting to different virtual characters or social robots. At the moment, we have compatible behavior realizers for a virtual character called "PseudoBot" and the Reeti robot ${ }^{2}$, both of which can be seen in figure 1. Another behavior realizer for the NAO $\operatorname{robot}^{3}$ is in progress, and we also plan to connect our system to more realistic virtual humans in the future.

\subsection{Shared Information Board}

The Shared Information Board collects the semantic content of each utterance spoken by any participant. This information is written to the board as soon as the "Minimum Necessary Information" (MNI for short) has been transmitted, which Chao [3] defined as the part of a communicative action after which a human would feel confident about its meaning. For example, this could be the name of a referenced object or the verb for a requested action. Therefore, the end of the MNI marks the earliest point in time at which the interlocutor's response will begin. For simple scenarios, this information can be marked by hand. We did so by inserting bookmarks into the text-to-speech commands for both agents which will trigger the addition of the MNI to the Shared Information Board.

${ }^{1}$ Examples for the generated behavior can be seen here: https://www.youtube.com/ playlist?list=PLAJ5ZtqkzFRtaO_kK9qPKvjxjzMWawBql

${ }^{2}$ http://www.reeti.fr

${ }^{3}$ https://www.softbankrobotics.com

\subsection{Influence Diagram}

An influence diagram is a Bayesian network with added nodes for decisions and their utilities. We use these utilities to model interaction goals and events that affect them, while their magnitude expresses the goals' priorities depending on the context.

The agent's attitude towards the user is modeled as chance nodes in the Bayesian network, based on psychological findings about the relationship between the Big Five personality factors and the Interpersonal Circumplex [5, 7]. This allows us to either configure the agent's personality from which the attitude towards the interlocutor is derived, or override this default interpersonal stance with situational information, such as the relative dominance based on the agent's task-related role.

The conversational role - speaker or listener - is represented by one chance node per participant. While the agent's intentions are known to the system, the (simulated) user's intended role can only be inferred from their observed behavior parameters, such as their voice activity and its duration. The influence diagram is updated with every new observation to reflect the knowledge about both participants. Whenever the dialog manager proposes a communicative action, it is delayed until the expected utility for executing it exceeds that of waiting. Vice versa, it is aborted when waiting becomes more lucrative.

Our current model covers only one single interaction goal, which is to exert control over the conversation. We chose this goal because the effect of dominance on turn-taking is already well documented in the literature $[2,8,9]$. The utilities for this goal were chosen based on those existing findings about how dominant a character's speech timing is perceived. Key combinations between situational variables were assigned utilities of 1.0 or -1.0 and the remaining values were inter- and extrapolated for all possible combinations of the agent's own role, the other party's role, the other party's speech or silence duration and the dominance expressed by the agent.

\section{REFERENCES}

[1] Dan Bohus and Eric Horvitz. 2011. Decisions about turns in multiparty conversation: from perception to action. In Proceedings of the 13th international conference on multimodal interfaces. ACM, 153-160.

[2] Angelo Cafaro, Nadine Glas, and Catherine Pelachaud. 2016. The effects of interrupting behavior on interpersonal attitude and engagement in dyadic interactions. In Proceedings of the 2016 International Conference on Autonomous Agents \& Multiagent Systems. International Foundation for Autonomous Agents and Multiagent Systems, 911-920.

[3] Crystal Chao. 2015. Timing multimodal turn-taking in human-robot cooperative activity. Ph.D. Dissertation. Georgia Institute of Technology.

[4] Cristina Conati. 2013. Virtual Butler: What Can We Learn from Adaptive User Interfaces? In Your Virtual Butler, Robert Trappl (Ed.). Lecture Notes in Computer Science, Vol. 7407. Springer Berlin Heidelberg, 29-41.

[5] Colin G. DeYoung, Yanna J. Weisberg, Lena C. Quilty, and Jordan B. Peterson. 2013. Unifying the Aspects of the Big Five, the Interpersonal Circumplex, and Trait Affiliation. Fournal of Personality 81, 5 (2013), 465-475.

[6] Julia A. Goldberg. 1990. Interrupting the discourse on interruptions: An analysis in terms of relationally neutral, power- and rapport-oriented acts. Fournal of Pragmatics 14, 6 (1990), 883 - 903.

[7] Robert R McCrae and Paul T Costa. 1989. The structure of interpersonal traits: Wiggins's circumplex and the five-factor model. Fournal of personality and social psychology 56, 4 (1989), 586.

[8] William T. Rogers and Stanley S. Jones. 1975. Effects Of Dominance Tendencies On Floor Holding And Interruption Behavior In Dyadic Interaction. Human Communication Research 1, 2 (1975), 113-122.

[9] Mark ter Maat, Khiet Phuong Truong, and Dirk K. J. Heylen. 2011. How Agents' Turn-Taking Strategies Influence Impressions and Response Behaviors. Presence: Teleoperators and Virtual Environments 20, 5 (Oct. 2011), 412-430. 